\title{
REPERCUSSÃO NA QUALIDADE DE VIDA E AUTOIMAGEM NA REALIZAÇÃO DA TÉCNICA DE MICROPIGMENTAÇÃO EM PACIENTES MASTECTOMIZADAS: UMA REVISÃO DE LITERATURA
}

\author{
Tamires Fernanda da Silva ${ }^{1}$ \\ Dayane Karoline G. D. de Araújo ${ }^{2}$
}

\section{RESUMO}

Também denominada maquiagem definitiva, a micropigmentação é uma técnica que ficou conhecida nos últimos anos. A remoção dos seios e as terapias adjuvantes contribuem para o desenvolvimento de complicações físicas e transtornos psicológicos que podem influenciar negativamente na qualidade de vida das mulheres. Este trabalho visa avaliar, através de uma revisão de literatura, as complicações na qualidade de vida das pacientes acometidas pelo câncer de mama e repercussões da autoimagem após a realização da técnica de micropigmentação em pacientes mastectomizadas. Foi realizada uma busca nas bases de dados eletrônicas MEDLINE (PUBMED), SCIELO E LILACS sem restrição de idiomas. Descritores estavam de acordo com o Medical Subject Headings (MeSH) e do Descritor em Ciências da Saúde (DeCS). Diante dos resultados apresentados, foi possível concluir que a técnica traz qualidade de vida e satisfação às pacientes.

Palavras-chave: Cancêr de mama. Micropigmentação. Autoimagem. Qualidade de vida.

Data de submissão: $23 / 04 / 2020$

Data de aprovação: $22 / 05 / 2020$

\section{INTRODUÇÃO}

O câncer de mama representa a principal causa de morte por câncer em mulheres brasileiras e, em nível mundial, cede o lugar apenas para o câncer de pulmão, representando um grande problema de saúde pública em todo o mundo (PAULINELLI et al, 2003).

O Brasil tem acompanhado as altas taxas de incidência e mortalidade de câncer de mama dos países desenvolvidos, porém as medidas necessárias à prevenção, ao diagnóstico e ao controle da doença não têm sofrido o mesmo crescimento. Foi estimado para o Brasil 
59.700 novos casos de câncer de mama para cada ano do biênio 2018-2019, com um risco estimado de 56,33 casos a cada 100 mil mulheres e uma sobrevida mundial de $61 \%$ após cinco anos. (BRASIL, 2008).

O câncer de mama é um dos mais temidos pelas mulheres devido a sua alta frequência e efeitos psicológicos, tais como alterações da imagem corporal, perda da feminilidade, medo de recidivas, ansiedade, dor e baixa autoestima, causando assim um impacto na vida dessas mulheres (FITOUSSI et al, 2008). Os principais sinais e sintomas de câncer de mama são nódulos na mama e/ou axila, dor mamária e alterações da pele que recobre a mama, como abaulamentos ou retrações com aspecto semelhante à casca de laranja.

Os principais fatores de risco para o desenvolvimento de câncer de mama relacionam-se com a idade avançada, características reprodutivas, história familiar e pessoal, hábitos de vida e influências ambientais. As características reprodutivas de risco se dão porque a doença é estrogênio-dependente e compreende a menarca precoce (aos 11 anos ou menos), a menopausa tardia (aos 55 anos ou mais), a primeira gestação após os 30 anos e a nuliparidade. (FITOUSSI et al, 2008).

Segundo Jammal et al (2008), o controle dessa doença se dá através da detecção precoce, na qual a lesão se restringe ao parênquima mamário, com um tamanho de no máximo três centímetros, permitindo o uso de recursos terapêuticos menos mutiladores e maior possibilidade de cura. Conforme dados do Ministério da Saúde (2008), os meios mais eficazes para a detecção precoce de câncer de mama são o exame clínico de mamas (ECM) e a mamografia, pois o autoexame de mamas (AEM) detecta a doença geralmente em estágio avançado, sendo responsável por cerca de $80 \%$ das descobertas de cânceres de mama.

A mastectomia tem por objetivo promover o controle local, com a remoção mecânica de todas as células malignas presentes junto ao câncer primário. Dentre as mastectomias, podemos destacar a radical modificada, que consiste na extirpação da mama e esvaziamento axilar radical, preservando o músculo peitoral maior, com ou sem preservação do peitoral menor. A mama representa o feminino para a mulher e a remoção da mesma provoca efeitos físicos, psicossociais, sexuais e emocionais que irão afetar diretamente a qualidade de vida dessas pacientes, (TAKEUTI et al, 2009). É considerada a forma mais eficaz para o tratamento do câncer de mama, pois as chances de sobrevivência da mulher são maiores, apesar de causar alguns incômodos como complicações físicas e transtornos mentais que implicam diretamente de forma negativa na vida da paciente. (OLIVEIRA et al, 2010).

Estudos realizados indicam que, ao receber o diagnóstico de câncer de mama, a mulher começa a enfrentar um momento difícil em sua vida, passando a vivenciar três etapas 
diferentes e complexas. A primeira refere-se ao diagnóstico de estar com câncer, palavra carregada de sentimentos negativos em nossa sociedade. A segunda é a realização de um tratamento muito longo e agressivo, muitas vezes com a necessidade de retirada parcial ou total da mama para restabelecimento da saúde. A terceira trata da aceitação de um corpo marcado e a convivência com essa imagem. (OLIVEIRA et al, 2010).

A mastectomia é um procedimento agressivo que provoca alterações emocionais, sociais e sexuais na mulher, podendo apresentar lesões musculares, diminuição ou perda da amplitude de movimento, alterações de posturas, dores, diminuição ou perda da capacidade funcional e linfedema do braço homolateral à cirurgia. (SILVA et al, 2014).

A micropigmentação é uma das técnicas que ficou bem conhecida dentro da estética nos últimos anos. Também denominada de maquiagem definitiva, esta técnica pode ser realizada em sobrancelhas, contornos dos lábios e dos olhos, e na reconstrução da aréola mamária após mastectomia, entre outros (MACHADO et al, 2014). É definida como a implantação de pigmentos (corantes) na camada subepidérmica da pele com o auxílio de um dermógrafo e agulhas apropriadas. Trata-se de uma técnica de permanência temporária da pigmentação, variando de 10 a 18 meses, necessitando de retoques para garantir a adequação com a cor da pele. A maioria das mulheres, após finalizarem a micropigmentação na aréola, geralmente apresentam uma melhoria da forma como se veem, tendo uma visão mais positiva de sua autoimagem.

A média de permanência do pigmento pode variar de cinco a quinze anos, dependendo da técnica empregada, do tipo e do grupo de agulhas usadas durante a pigmentação, da base e saturação dos pigmentos aplicados e dos hábitos de vida da paciente. (TAKEUTI et al, 2009).

A técnica de dermopigmentação devolve o bem-estar e melhora a qualidade de vida das pacientes. Ainda ameniza o desconforto da aparência estética, devolvendo a essas mulheres autoestima e uma nova chance de recomeço. (SANDERSON et al, 2009).

Cerigatto (2013) observou um grupo de mulheres que realizaram a dermomicropigmentação no complexo da aréola mamilar e identificou, do ponto de vista estético e psicológico, uma visão mais positiva de suas autoimagens, desfazendo crenças irracionais em torno da perda da beleza e da sensualidade depois de terem sido mastectomizadas. Nas que ficaram com sequelas de cicatrizes e queloides após mastopexia e redução mamária ocorreu melhora da autoestima, devolvendo, segundo os autores, parte da feminilidade da mulher. 
Este trabalho visa avaliar, através de uma revisão de literatura, as complicações na qualidade de vida das pacientes acometidas pelo câncer de mama e repercussões da autoimagem após a realização da técnica de micropigmentação.

\section{METODOLOGIA}

Foi realizada uma revisão de literatura através de buscas nas bases de dados eletrônicas MEDLINE (PUBMED), SciELO e LILACS sem restrição de idioma. Através do Medical Subject Headings (MeSH) e do Descritor em Ciências da Saúde (DeCS) foram extraídos os seguintes descritores para seleção dos artigos: Breast Neoplasms, Mastectomy, Pigmentation; utilizando o operador booleano "AND”. CHAVES DE BUSCA: -PUBMED: (("Breast Neoplasms Modalities"[Mesh]) AND "Mastectomy"[Mesh]) AND "Pigmentation "[Mesh]; SciELO: (Câncer de Mama) AND (Mastectomia) AND (Micropigmentação); LILACS: Câncer de Mama [Palavras] and Mastectomia [Palavras] and Micropigmentação [Palavras].

Foram selecionados os artigos no período de 2008 a 2019, sem restrição de idioma, limitando-se a estudos em humanos. Além disso, foi realizada a procura de artigos que poderiam ser selecionados para a pesquisa, a partir das referências bibliográficas dos artigos selecionados.

Foram excluídos cartas, resumos, cartazes, editoriais, e trabalhos que avaliavam concomitantemente outros tipos de micropigmentação ou que não abordassem a qualidade de vida das mastectomizadas. Os seguintes critérios de inclusão foram aplicados: (1) Mulheres que passaram pelo processo da mastectomia; (2) Avaliação da qualidade de vida após a reconstrução da mama. Depois de incluídos, foi realizada uma revisão bibliográfica dos resultados encontrados através da descrição dos estudos e características do protocolo.

Dos 17 artigos encontrados (PUBMED 2, LILACS 3, SCIELO 4 e Google Acadêmico (8), 5 deles foram excluídos pelo título/resumo, pois abordavam outros tipos de micropigmentação. Dos doze artigos restantes dois foram excluídos por não preencherem os critérios de inclusão. Dez estudos foram selecionados para a análise qualitativa, sendo submetidos a uma análise integral com aprofundamento de seus conteúdos, conforme quadro a seguir. 
Quadro 1 - Análise integral com aprofundamento de conteúdo

\begin{tabular}{|c|c|c|c|c|c|}
\hline $\begin{array}{l}\text { AUIOR } \\
(\text { ANO) }\end{array}$ & 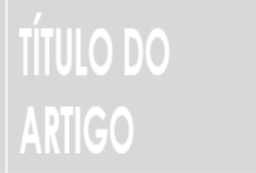 & $\begin{array}{l}\text { INPO DO } \\
\text { ESIODO }\end{array}$ & OBN=N/VOS & RESUIADOS & CONCLUSÃO \\
\hline $\begin{array}{l}\text { Cammarota } \\
\text { et al } 2019\end{array}$ & $\begin{array}{l}\text { Qualidade de vidae } \\
\text { resultado estéitico } \\
\text { após massectomia e } \\
\text { reconstrução } \\
\text { mamária }\end{array}$ & $\begin{array}{l}\text { Observacional } \\
\text { longitudinal }\end{array}$ & $\begin{array}{l}\text { Apresentar a importância } \\
\text { da micropigmentaçço } \\
\text { para recuperaçaco da } \\
\text { autoesima em pacientes } \\
\text { submetidas à } \\
\text { mastectomia }\end{array}$ & 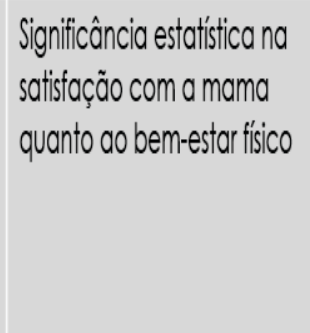 & $\begin{array}{l}\text { A qualidade de vida no } \\
\text { período posterior a } \\
\text { reconstrução mamária é } \\
\text { superior em relaçãa a do } \\
\text { anterior }\end{array}$ \\
\hline $\begin{array}{l}\text { Machado et al } \\
2014\end{array}$ & $\begin{array}{l}\text { Dermopigmentação } \\
\text { cutânea em } \\
\text { pacientes } \\
\text { mostectomizadas }\end{array}$ & $\begin{array}{l}\text { Revisiõo } \\
\text { sistemática }\end{array}$ & $\begin{array}{l}\text { Avaliar a importância da } \\
\text { dermopigmentação em } \\
\text { pacientes submetidaa } \\
\text { mastectomia }\end{array}$ & $\begin{array}{l}\text { O câncer de mama e a } \\
\text { cirurgia causam grande } \\
\text { temor entre as muhheres, } \\
\text { provocam alteraçóoes } \\
\text { psicológicas, afeta a } \\
\text { percepção de sexualidade } \\
\text { e imagem pessoal }\end{array}$ & $\begin{array}{l}\text { Tem como objefivo a } \\
\text { colorir a epiderme, dando } \\
\text { um aspecto natural } \\
\text { reconstrução da mama } \\
\text { favorecendo a autroesilima } \\
\text { e qualidade de vida }\end{array}$ \\
\hline $\begin{array}{l}\text { Silva et al } \\
2014\end{array}$ & $\begin{array}{l}\text { Qualidade de vida } \\
\text { pós mastectomia e } \\
\text { sua relaçāo com a } \\
\text { força muscular de } \\
\text { MMSs }\end{array}$ & $\begin{array}{l}\text { Estudo } \\
\text { transversal }\end{array}$ & $\begin{array}{l}\text { Identificara qualidade de } \\
\text { vida de mulheres } \\
\text { mastectomizadas } \\
\text { relacionar os resultados } \\
\text { com a força e traçar um } \\
\text { peril sociofuncional }\end{array}$ & $\begin{array}{l}\text { A qualidade de vida } \\
\text { estava regular, as principais } \\
\text { alteraçōes foram na escala } \\
\text { emocional, cognitiva e } \\
\text { sexual }\end{array}$ & $\begin{array}{l}\text { Apontam impacto do } \\
\text { câncer de mama em } \\
\text { relação a qualidade de } \\
\text { vida em especial nos } \\
\text { aspectos sociais de suas } \\
\text { vidas }\end{array}$ \\
\hline $\begin{array}{l}\text { Cesar et al } \\
2013\end{array}$ & $\begin{array}{l}\text { Qualidade de vida } \\
\text { em pacientes } \\
\text { mostectomizadas em } \\
\text { reabilitaçáo } \\
\text { oncologica }\end{array}$ & $\begin{array}{l}\text { Estudo de } \\
\text { caso }\end{array}$ & $\begin{array}{l}\text { Avaliar a Qualidade de } \\
\text { vida em pacientes } \\
\text { mastectomizadas em } \\
\text { reabilitração }\end{array}$ & Abordagem multidisciplinar & $\begin{array}{l}\text { Concluiu-se que a terapia } \\
\text { de grupo contribuiv em } \\
\text { todos os sentidos para } \\
\text { melhora de vida dessas } \\
\text { pacientes }\end{array}$ \\
\hline $\begin{array}{l}\text { Oliveira et al } \\
2010\end{array}$ & $\begin{array}{l}\text { Efeitio da } \\
\text { reconstrução } \\
\text { mamári imediata } \\
\text { sobre a qualidade de } \\
\text { vida }\end{array}$ & $\begin{array}{l}\text { Estudo } \\
\text { transsersal }\end{array}$ & $\begin{array}{l}\text { Avaliar propectivamente } \\
\text { os ééitios da reconstrução } \\
\text { mamária imediata sobre } \\
\text { a qualidade de vida de } \\
\text { muhheres } \\
\text { mastectomizaddas }\end{array}$ & $\begin{array}{l}\text { Observou-se redução no } \\
\text { primeiro mês, com } \\
\text { recuperação significativa } \\
\text { após bmeses }\end{array}$ & $\begin{array}{l}\text { A reconstrução mamária } \\
\text { imediata é benéfica para } \\
\text { aspectos psicológicos e na } \\
\text { qualidade de vida }\end{array}$ \\
\hline
\end{tabular}

Fonte: as autoras 


\section{DISCUSSÃO}

O propósito deste trabalho foi avaliar, através de uma revisão de literatura, as complicações na qualidade de vida das pacientes acometidas pelo câncer de mama, apontando as complicações na qualidade de vida dessas pacientes submetidas à reconstrução da mama e repercussões da autoimagem após a realização da técnica de micropigmentação.

Cezar \& Nascimento (2013) realizaram um estudo de caso em que foi possível concluir que no início e após cinco meses de atendimento foi feita a avaliação da qualidade de vida aplicada por meio do questionário SF 36 e através da análise descritiva dos dados. Concluiuse que a terapia de grupo contribui para a melhora da qualidade de vida dessas pacientes. A abordagem multidisciplinar na atenção de pacientes com câncer de mama determina melhores resultados no seu controle. A convivência de grupos com problemas semelhantes proporciona experiências que podem desenvolver um clima de muito valor terapêutico.

No estudo de Cammarota et al (2019) foram selecionadas 74 pacientes que foram submetidas à reconstrução mamária com implantes, sendo 59 com prótese de silicone e 15 com expansor. A idade das pacientes variou de 24 a 81 anos. Para a avaliação da qualidade de vida das pacientes foi utilizado o BREAST-Q ${ }^{\circledR}$, um questionário validado internacionalmente para o desenvolvimento de escalas de avaliação da qualidade de vida relacionada à reconstrução mamária sob a perspectiva do paciente.

A maioria das pacientes foi submetida à reconstrução imediata $(95,94 \%)$. Foi possível constatar que a reconstrução de mama, apesar de todas as complicações inerentes ao processo, traz qualidade de vida e satisfação às pacientes. Em todos os domínios analisados (satisfação com a mama, bem-estar psicossocial, bem-estar físico e bem-estar sexual) houve maior pontuação no pós-reconstrução em comparação com o pré-reconstrução. Segundo relatos da literatura, a maioria das mulheres está optando por essa forma de reconstrução mamária na tentativa de reduzir os sentimentos negativos desencadeados pela doença e seu tratamento.

Além das relações entre as pacientes mastectomizadas e as pessoas do seu convívio, é de crucial importância o papel da equipe multidisciplinar na tentativa de resgatar o conceito que a mulher mastectomizada tem de si mesma. Daí a necessidade de uma reconstrução, devolvendo a essa mulher a vontade de viver e a autoestima. É preciso preocupar-se não apenas com a recuperação do câncer, como também com a reabilitação global. (MACHADO et al, 2014). 


\section{CONCLUSÃO}

Foi possível constatar, por intermédio deste trabalho, que a reconstrução de mama, apesar de todas as complicações inerentes ao processo, traz qualidade de vida e satisfação às pacientes. Também foi possível perceber que a maioria das pacientes está optando por essa forma de reconstrução mamária, uma vez que esta técnica visa a reduzir os sentimentos negativos desencadeados pela doença e pelo tratamento.

Diante dos resultados apresentados foi possível concluir que a micropigmentação é referida como sendo uma forma alternativa para melhorar o aspecto estético em pacientes mastectomizadas, trazendo de volta a autoestima e a qualidade de vida para esse grupo de pacientes.

\section{REFERÊNCIAS}

TAKEUTI, Paula et al. Avaliação da qualidade de vida de mulheres mastectomizadas inseridas em um programa de fisioterapia aquática. 2009. Disponível em: https://ojs.unesp.br/index.php/revista_proex/article/view/1008/923.Acesso em: 20 mar. 2019.

BRASIL. Ministério da Saúde. Instituto Nacional do Câncer. Ações de enfermagem para o controle do câncer. 3. ed. Rio de Janeiro: INCA; 2008.

CAMMAROTA, M. C. et al. Qualidade de vida e resultado estético após mastectomia e reconstrução mamária, Revista Bras. de Cirurgia Plástica. 2019. Vol. 34. Disponível em: http://rbcp.org.br/details/2344/pt-BR/qualidade-de-vida-e-resultadoestetico-aposmastectomia-e-reconstrucao-mamaria. Acesso em: 12 maio 2019.

CERIGATTO, M. et al. Micropigmentação facilita dia a dia da mulher moderna. Disponível em: http://www.jcdigital.com.br/flip/Edicoes/15047\%3D26-062011/56.PDF. Acesso em: 17 mar. 2019. 
CEZAR, K.; NASCIMENTO, A. P. C. Qualidade de vida de pacientes pósmastectomizadas. Cuiabá: Universidade de Cuiabá, 2013. Disponível em: http://www.unifra.br/eventos/forumfisio/Trabalhos/5088.pdf. Acesso em: 15 nov. 2018.

FITOUSSI, A. D. et al. Nipple and areola reconstruction by tattooing, $\mathbf{F}$ and $\mathbf{Z}$ flaps. Disponível em: https://www.ncbi.nlm.nih.gov/pubmed/18387725._Acesso em 21 mar. 2019.

JAMMAL, M. P.; MACHADO, A. R. M.; RODRIGUES, L. R. Fisioterapia na reabilitação de mulheres operadas por câncer de mama. O Mundo da Saúde. São Paulo: 2008. Disponível em: https://http://files.sandrabarbosa.webnode.com.br/200000045396a13a622/12_Fisioterapia\%20cancer\%20de\%20mama.pdf. Acesso em: 23 nov. 2019.

MACHADO, F. A. Dermopigmentação cutânea em pacientes mastectomizadas. Revista Eletrônica de Saúde e Ciência, Goiânia, v. 4, n. 2, 2014. Disponível em: http://www.rescceaf.com.br/vol2/n2/dermopigmentação\%20pags\%2055\%20a\%2068.pdf. Acesso em: 19 nov. 2018.

MARTINS, M. C.; MEJIA, D. P. M.; AZEVEDO, A. M. A Micropigmentação Paramédica Areolar Pós-Mastectomia. Portal bio cursos. Disponível em: http://portalbiocursos.com.br/ohs/data/docs/199/13A_MicropigmentaYYo_ParamYdica_Areo lar_PYs-Mastectomia.pdf. Acesso em: 11 mar. 2019.

OLIVEIRA, R. R.; MORAIS, S. S.; SARIAN, L. O. Efeito da reconstrução mamária imediata sobre a qualidade de vida. Revista Bras. Ginecol. Obstet. 2010. Vol.32. n.12. Disponível em: http://www.scielo.br/scielo.php?script=sci_arttext\&pid=S0100-72032010001200007. Acesso em mar 2019.

PAULINELLI, R. R. et al. A situação do câncer de mama em Goiás, no Brasil e no mundo: tendências atuais para a incidência e a mortalidade. Revista Bras. Saúde Mater. Infant. 2003. 3(1): 17-24. Disponível em: https://www.scielo.br/scielo.php?pid=S151938292003000100004\&script=sci_arttext\&tlng=pt. Acesso em: 29 mar. 2019. 
SILVA, S. H.; KOETZ, L. C. E.; SEHNEM, E.; GRAVE, M. T. Q. Qualidade de vida pósmastectomia e sua relação com a força muscular de membro superior. 2014. Fisioterapia e pesquisa. Disponível em: https://www.scielo.br/scielo.php?pid=S180929502014000200180\&script=sci_arttext\&tlng=pt. Acesso em: 15 maio 2019.

SANDERSON, B. F. et al. Dermopigmentação uma alternativa estética e reparadora. ULBRA, Santa Maria, 2009. 